\title{
SÍNDROME METABÓLICO Y DISFUNCIÓN ERÉCTIL
}

\author{
Remigio Vela Navarrete, Jesús Gómez Muñoz, Leticia López Martín, Juan Vicente García \\ Cardoso y Carmen González Enguita.
}

Servicio de Urología y Unidad de Transplante renal. Fundación Jiménez Díaz. Universidad Autónoma de Madrid. Madrid. España.

\begin{abstract}
Resumen.- OBJETIVO: Puesta al día de un síndrome que en los pasados años ha ido incrementando las patologías coincidentes en el mismo, tales como obesidad, hipertensión, hipercolesterolemia, diabetes tipo II, con la reciente adición de la disfunción erectil y la androgenodeficiencia

MÉTODO: Revisión global de la literatura tomando como referencia las últimas aportaciones y muy especialmente las debidas al profesor M. Serrano Ríos, de Madrid, y su grupo

RESULTADOS: El síndrome metabólico parece actualmente consolidado como terminó universalmente aceptado, a pesar de su complejo itinerario semántico y nosologico. La inclusión de la disfunción erectil y la androgenodeficiencia le da más solidez patogénica y
\end{abstract}

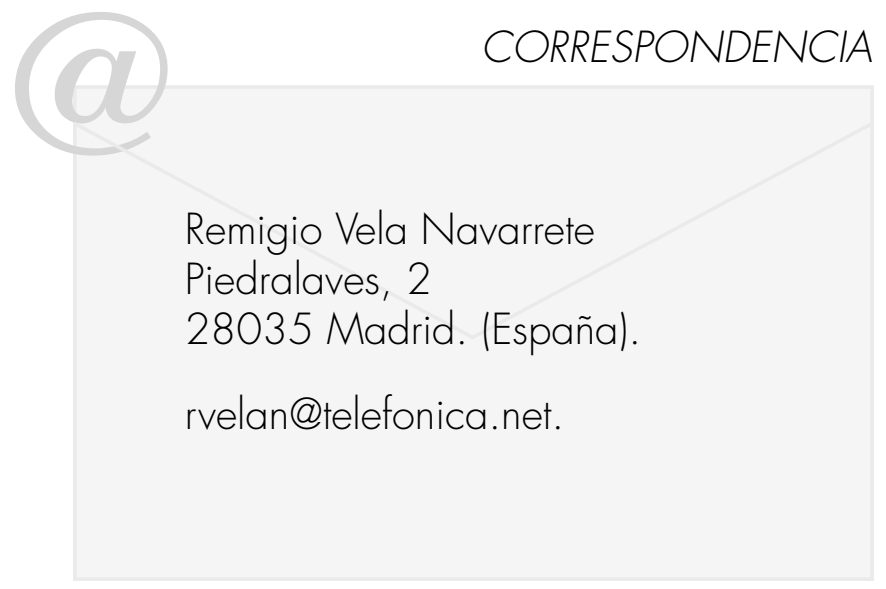

aproxima más al terreno profesional de la endocrinología a dos procesos prioritariamente urológicos. El urólogo recibe a su vez una nueva perspectiva de procesos que son inexcusablemente propios y a los que ha de atender con mayor amplitud exploratoria, analítica y terapéutica

CONCLUSIÓNES: El síndrome metabólico puede ser reconocido en la consulta urológica con más frecuencia de lo sospechado. El urólogo queda obligado, en este proceso típicamente médico, a ejercer con más dedicación y amplitud el compromiso médico de su especialidad médico quirúrgica.

Palabras clave: Síndrome metabólico. Disfunción eréctil. Androgenodeficiencia.

Summary.- OBJECTIVES: To update a syndrome that has increased the number of pathologies included such as obesity, hypertension, hypercholesterolemia, type II diabetes mellitus, and the recent addition of erectile dysfunction and androgen deficiency

METHODS: Global review of bibliography taking the last articles as a reference and mainly those from Prof. M. Serrano Rios and his group in Madrid.

RESULTS: Metabolic syndrome seems to be consolidated as a universally accepted term, despite its complex semantic and gnoseologic itinerary. The inclusion of erectile dysfunction and androgen deficiency gives more pathogenic solidity and makes the professional field of endocrinology closer to two mainly urological processes.

The urologist has a new perspective of processes that are of his own, that he has to take care of in a comprehensive manner, with physical examination, blood tests and therapy. 
CONCLUSIONS: Metabolic syndrome may be more frequently than suspected recognized in the urologist office. Urologists are compelled, in this typically medical process, to exercise with more dedication and fuIlness the medical compromise of our medical-surgical specialty.

Keywords: Metabolic sindrome. Erectile dysfunction. Androgen deficiency.

\section{INTRODUCCIÓN}

El síndrome metabólico (SMet) ni es solo un síndrome ni es exclusivamente metabólico, pero la construcción de este concepto nosológico, aún incompleto, a lo largo del tiempo, ha tenido la virtud de hacernos comprender que muchos de los procesos asociados a la obesidad (ahora adiposidad), como la diabetes tipo 2, hipertensión o dislipidemia, no son simples complicaciones de la obesidad, sino que constituyen por sí mismos trastornos y disfunciones que coinciden patogénicamente con este proceso, el más llamativo, pero no el único protagonista de este trastorno metabólico (1-3). Este mal llamado síndrome, pero ya con nombre consagrado (Tabla I), es fruto de la observación clínica que a lo largo de estos últimos años ha comprobado, primero con la simple observación y después con estudios epidemiológicos precisos (3-5), que muchos de estos procesos enumerados en la Tabla I, y a los que nuevamente se van añadiendo otros, "nunca vienen solos", sino acompañados, unas veces dominando unos y otras veces otros $(6,7)$. En definitiva, el síndrome metabólico es un "racimo" de enfermedades, trastornos analíticos, disfunciones de muy variados órganos (Figura 1) a los que hay que acercarse con la sospecha de que tienen un nexo patogénico común, que no representan una enfermedad especifica de órgano sino la coincidencia de trastornos sistémicos cuyas repercusiones somáticas, analíticas y disfuncionales hay que entenderlas de manera integral e integrada.

\section{¿Por qué es importante el síndrome metabólico para el urólogo?}

Por su ejemplaridad integradora. El urólogo en sus actividades médicas está fundamentalmente habituado a tratar patologías de órganos. De este modo, cuando enfrentado a la patogenia de la disfunción eréctil ha estado durante muchos años convencido de que la respuesta a este trastorno estaba en las investigaciones anatómicas y funcionales fundamentalmente relacionadas con el pene. Hoy día sabemos que un gran porcentaje de estas disfuncio- nes están relacionadas con problemas sistémicos de expresión e intensidad variable y que donde hay que buscar el fundamento patogénico de este trastorno es en el sistema vascular, en el endotelio, de estos individuos. De igual modo, conceptualmente el síndrome metabólico ha demostrado que más allá de la consulta específica de estos pacientes por obesidad, diabetes, hipertensión, etc. existen otros trastornos cada día mejor conocidos, que justifican su coincidencia y que advierten de la existencia de alteraciones básicas comunes. En definitiva, si el urólogo quiere hacer una medicina más cabal, integradora y no parcelar, como recomiendan las nuevas tendencias y las campañas más recientes (envejecimiento activo, longevidad activa y saludable, el urólogo como generalista del varón añoso o del varón a cualquier edad, siguiendo el ejemplo de los ginecólogos) tendrá necesariamente que familiarizarse con todos los procesos enumerados, que constituyen el síndrome metabólico más amplio, para reconocer estos procesos en su consulta y no ignorar hasta dónde puede llegar en sus actitudes diagnósticas y terapéuticas, reclamando o no la ayuda de otras especialidades en función de la amplitud y gravedad de los procesos que detecta. Téngase en cuenta que la causa más común de consulta médica de cualquier tipo en los va-

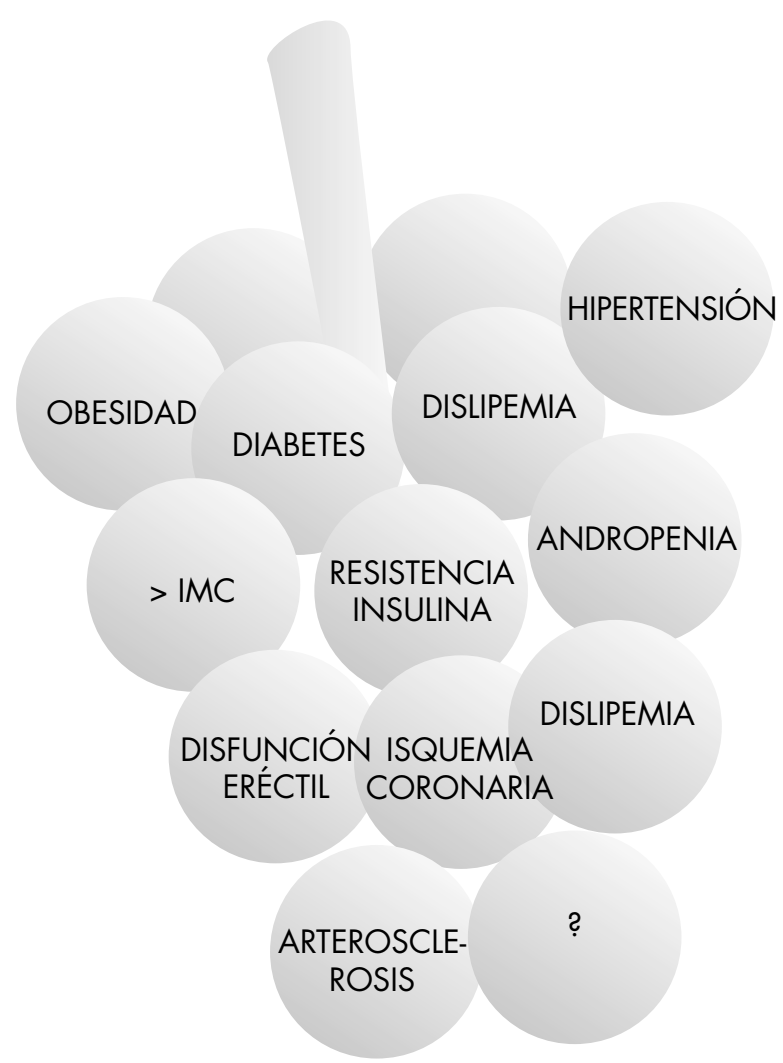

FIGURA 1. Síndrome metabólico, más que un síndrome, racimo de enfermedades, disfunciones y trastornos analíticos. 


\section{TABLA I. DEFINICIÓN DE SÍNDROME METABÓLICO SEGÚN LA ORGANIZACIÓN MUNDIAL DE LA SALUD,} BASADA EN LOS SIGUIENTES CRITERIOS DIAGNÓSTICOS.

\section{Regulación alterada de la glucosa, diabetes y o resistencia a la insulina}

\section{Coincidente con dos o más de los siguientes trastornos (en varones):}

- Tensión arterial elevada (mayor o igual 140/90 mmhg)

- Triglicéridos plasmáticos elevados (mayor o igual 1,7mmol/L o $150 \mathrm{mg} / \mathrm{dl}$ )

- Hipercolesterolemia (mayor o igual 200 mg/dl)

- HDL bajo (inferior a 0,9 mmol/L o $35 \mathrm{mg} / \mathrm{dl}$ )

- Obesidad central (relación cintura cadera mayor 0, 90) y/o índice de masa corporal (IMC) mayor de $30 \mathrm{~kg} / \mathrm{m}$

- Microalbuminuria (mayor $30 \mathrm{mg} / \mathrm{kg}$ )

rones mayores de 50 años es al urólogo por trastornos miccionales y en consecuencia el urólogo se ha convertido en el más importante actor sanitario del diagnóstico precoz de numerosas patologías y en un referente fundamental como proveedor de estrategias preventivas en el varón, función que sin duda comparte con el generalista aunque no de manera tan eficiente en todos los sistemas de salud europeos.

\section{Cómo evaluar un paciente con Síndrome Metabólico (¿Sospechado?)}

Como señalábamos, aunque es conveniente insistir, el urólogo es en la actualidad el especialista más visitado por los varones mayores de 50 años por síntomas urinarios o simplemente para confirmar su salud prostática. Es, por lo tanto, en este grupo de población donde el urólogo debe ejercer de una manera más rigurosa sus posibilidades diagnósticas pre- coces y donde el ejercicio de su práctica clínica se ve actualmente más comprometido. Otros pacientes llegarán a su consulta ya con diagnósticos previos de sus generalistas para consultas y recomendaciones de patologías específicas. Ante la evidencia de que el sobrepeso, la disfunción eréctil, la andropenia, etc. forman parte del síndrome metabólico e incluso en algunas circunstancias constituyen un heraldo de patología cardiovascular severa, pero preventiva, su valoración clínica no puede quedar reducida a una simple exploración de órganos. Por el contrario, es obligada una nueva actitud exploradora y por supuesto una nueva aptitud formativa. Quedan ahora plenamente justificadas las específicas responsabilidades en la investigación de la salud del varón, a cualquier edad, pero más precisamente en el varón añoso. Por lo tanto, la historia clínica debe ser completa, no reducida exclusivamente a sintomatología de órganos y muy especialmente detectando comorbilidades, aparentes u ocultas, pero sospechadas, e

\section{TABLA II. SÍNDROME METABÓLICO EVALUACIÓN UROLÓGICA.}

Examen físico: talla: peso: IMC: perímetro abdominal: tensión arterial: tacto rectal: examen genital

Analítica: Hemograma. Bioquímica clínica general: glucemia: Hbglioxilada: PSA total: Testosterona total: otros marcadores (microalbuminuria, marcadores de inflamación -adipocinas-, marcadores de lesión endotelial, SGHB)

Técnicas de imagen y función aparato urinario inferior: en relación con la patología urológica sospechada: orina residual; estudios urodinámicos o videourodinámicos; etc. 
indagar sobre tratamientos farmacológicos coincidentes. El examen físico no puede quedar reducido a un tacto prostático; las posibilidades actuales para determinar el peso y talla del individuo, su perímetro abdominal, su tensión arterial (aparatos simplificados), dentro o fuera de la consulta, son fácilmente asequibles. En cuanto a la analítica, es ahora exigible una mayor amplitud, si no está ya determinada por el generalista, que habitualmente incluye marcadores de función renal y función hepática, glucemia, perfil lipídico, así como PSA total, testosterona total, y otros. Es sorprendente como muchos pacientes diabéticos que vienen a consultar por disfunción eréctil, muchos de ellos con otras comorbilidades (hipertensión, sobrepeso, insuficiencia renal) no tienen una determinación de testosterona total como referencia mínima, pero fundamental, de su estatus androgénico. La oportunidad de otras determinaciones analíticas como la globulina transportadora de la hormona sexual(SHBG ) o estrógenos totales ha de confirmarse en el futuro.

En casos concretos, pueden existir complicaciones urológicas que necesiten estudios específicos, anatómicos y funcionales del aparato urinario inferior. El Síndrome Metabólico motiva con frecuencia empeoramiento del síndrome miccional por hiperactividad vesical o acontractilidad del detrusor, incluso con incontinencia, o empeoramiento sintomático de una HBP ya conocida, con infecciones urinarias recidivantes y a veces graves, además de la disfunción eréctil.

\section{Aspectos patogénicos}

Cómo señalado, se busca una referencia común, básica, que justifique este racimo de patologías coincidentes (8). La resistencia a la insulina se ha considerado la responsable de la mayor parte de los trastornos y en especial de la hiperglucemia, hipertensión arterial, aumento de la producción hepática de VLDL y triglicéridos y proliferación endotelial (9-13). La resistencia a la insulina se define como la incapacidad de la insulina para actuar sobre órganos dianas como el hígado, el músculo esquelético y el tejido adiposo, lo que supone la elevación de la glucemia e hiperinsulinemia secundaria. La causa por la que los tejidos no responden de manera adecuada al estímulo de la insulina permanece oscura pero se considera que la obesidad y la adiposidad abdominal tienen un protagonismo especial en este trastorno. Efectivamente, el sobrepeso y la obesidad están vinculados con diabetes, hipertensión y síndrome metabólico mucho más que otras condiciones físicas. Al tejido adiposo se le considera ahora como uno órgano endocrino capaz de sintetizar hormonas y proteínas de carácter inflamatorio conocidos como adipocinas, entre las que comienzan a tener una personalidad específica la resistina, la adiponectina y la leptina. Debemos recordar también que el tejido adiposo participa en la aromatización de la testosterona generando estrógenos. Sin embargo, no está muy claro aún cuál de las adipocinas mencionadas es la más activa en la lesión vascular y endotelial, fin último de muchos de estos enfermos, pero la paradoja de que a mayor cantidad de grasa, mayor cantidad de estrógenos, nos hace sospechar de la existencia de mecanismos autoreguladores y protectores que progresivamente serán mejor establecidos. Los trastornos genéticos y nutricionales causantes iniciales de estos procesos también están en estudio, pero por ahora no tienen una aplicación clínica fundamental en nuestra práctica habitual.

Los mecanismos implicados en el daño endotelial observados en individuos con síndrome metabólico tienen especial interés por su nexo con la disfunción eréctil. Está bien establecida la relación entre disfunción endotelial, resistencia a la insulina y cronicidad diabética. En estos pacientes están elevados los reactantes de fase aguda y algunas citoquinas inflamatorias que pueden ser producidas por el tejido adiposo. La lesión endotelial puede ser multifactorial y progresiva, manifestándose inicialmente por disfunciones de poco relieve clínico, a nivel coronario o peneano, no tan expresivas como para reconocerse en la clínica o motivar disfunción eréctil relevante, asociándose más tarde a patología vascular orgáni$\mathrm{ca}$, ateromatosa, ya que entre los elementos mencionados algunos contribuyen a disminuir la adhesión, quizás la leptina y el TNF, facilitando la aterotrombosis (14-16).

En la disfunción eréctil del síndrome metabólico, además de los factores mencionados seguramente tiene un protagonismo especial la andropenia, frecuentemente detectada en estos individuos. El mecanismo íntimo por el que la testosterona mejora la función endotelial implicada en la erección ha sido especialmente estudiado por el grupo de Traish (17) pero es conveniente recordar que la testosterona por sí misma tiene un efecto vaso dilatador que justificó en tiempos su uso en la isquemia coronaria (Vela Navarrete R. et al.2009; Lopez Farré A., Macaya C.) $(18,19)$.

\section{Aspectos terapéuticos}

¿Debe el urólogo tratar el síndrome metabólico? Este proceso mórbido sólo puede ser valorado y tratado desde una perspectiva integradora, no especializada, como la que corresponde a lo que 
tradicionalmente hemos calificado de internistas, o expertos en Medicina Interna. Como señalado previamente, el urólogo tiene un papel decisivo en el diagnóstico precoz de muchos de estos procesos y en su valoración inicial, pero también con notable frecuencia, los enfermos llegan a nuestra consulta ya diagnosticados y tratados, tomando antidiabéticos, hipotensores, estatinas, etc. y al urólogo sólo se le solicita colaboración en algún tema específico como prostatismo, disfunción eréctil, incontinencia, etc. En todas estas circunstancias el urólogo puede ser extremadamente útil no sólo para mejorar la calidad de vida del paciente mediante actuaciones correctoras de los síntomas, si no evitando interacciones farmacológicas inoportunas. Nos referimos aquí fundamentalmente a las relacionadas con la farmacología de la disfunción eréctil pero existen monografías recientes dedicadas a otros trastornos del aparato urinario coincidentes con la obesidad y el síndrome metabólico (Daneshgary, F. Symposium on urology complications of obesity and diabetes. J. Urol. 182-62 2009).

Las recomendaciones generales más comúnmente propuestas para moderar o disminuir la disfunción eréctil en pacientes con síndrome metabólico, seguramente ya propuestas por el generalista o el diabetólogo son: el abandono del tabaquismo; cambios en el estilo de vida con especial insistencia en el ejercicio físico; reducción del sobrepeso; cumplimiento estricto de las recomendaciones para el tratamiento de la diabetes. Quizás los aspectos específicos que afectan de una manera más concreta al urólogo pueden resumirse con las siguientes preguntas: ¿̇cuáles son los hipotensores, antidiabéticos, reguladores del colesterol, etc. que menos efecto tienen sobre la disfunción eréctile, ¿̇cuál es el mejor inhibidor de la fosfodiesterasa en los diabéticos?: ¿en qué pacientes se está recomendando el uso coincidente de testosterona e inhibidores de la PDE5?, ¿̇tiene algún beneficio el uso continuado, no a demanda, de los inhibidores de la PDE5?, etc. Todas estas preguntas demuestran algo concreto: el tratamiento de estos pacientes debe hacerse de manera individualizada apartándose o no, según los casos, de guías y protocolos que sustituyan incorrectamente la obligación de reflexionar sobre el paciente concreto.

Los hipotensores más recomendados en los pacientes con síndrome metabólico o diabetes, por su efecto protector sobre la función renal, son los IECA o los ARA-II. Sin embargo, sospechamos que tienen más efectos negativos sobre la función eréctil que otras alternativas. $(21,22)$

No existen datos para saber con precisión cuál es la mejor estatina en estos pacientes aunque
ATORVASTATINA continúa siendo la más recomendada (23). No es función del urólogo elegir el fármaco antidiabético más conveniente pero si aconsejar la visita al experto.

No existen por el momento trabajos específicamente orientados a demostrar si el uso continuado con inhibidores de la PDE5 pueden tener algún beneficio especial en los pacientes con síndrome metabólico o pueden sustituir a las estatinas. En los pacientes diabéticos, nuestra experiencia es que la respuesta más satisfactoria se consigue con VARDENAFILO a demanda.

El tratamiento con testosterona del Síndrome de Déficit de Testosterona, coincidente con el síndrome metabólico, parece ahora inexcusable. Un reciente estudio ha demostrado que el tratamiento continuado con testosterona en pacientes con síndrome metabólico, mejoró todos los marcadores del síndrome como la hipertensión, resistencia a insulina, obesidad abdominal y dislipidemia (24-26). La terapéutica sustitutiva androgénica sí constituye un compromiso inexcusable del urólogo que decidirá la mejor estrategia en función de objetivos y paciente. Además debe alertar de los efectos demoledores de la coincidencia de SM y androgenodeficiencia (27-29).

Pero el urólogo tiene ocasión de atender otro síndrome metabólico que a veces se motiva entre sus pacientes debido al uso continuado de regímenes de castración y, especialmente de regímenes de castración con análogos $\mathrm{LH}-\mathrm{RH}$. Los enfermos tratados con esta medicación por un período superior a los 6 meses, deben ser evaluados periódicamente, no solo recurriendo al comportamiento de su PSA, sino valorando otras funciones relacionadas con la deficiencia androgénica motivada por el tratamiento. Al menos, debe incluirse en la evaluación analítica, además de la testosterona, la glucemia, hemoglobina y perfil lipídico; y en la consulta, peso, talla y perímetro abdominal.

\section{BIBLIOGRAFÍA y LECTURAS RECOMENDADAS ( ${ }^{*}$ lectura de interés $y^{* *}$ lectura fundamental)}

1. WHO consultation: Definition, diagnosis and classification of diabetes mellitus and its complications. WHO/NCD/NCS/1999.2; 31-3.

**2. Serrano Ríos, M. Síndrome metabólico. Tiempos médicos $200966 \quad 245-48$

3. Alvarez EE, Ribas L, Serra L. Prevalencia del síndrome metabólico en la población de la Comunidad Canaria. Med Clin (Barc). 2003;120:172-4.

4. Alegría E, Cordero A, Grima A, Casasnovas JA, 
Laclaustra M, Luengo E, et al.. Prevalencia del síndrome metabólico en población laboral española: Registro MESYAS. Rev Esp Cardiol. 2005;58:797-806.

**5. Cordero A, Alegría E, León M. Prevalencia del síndrome metabólico. Rev Esp Cardiol. 2006;5:11-15.

**6. Yassin, A.A.; Saad, F.; Gooren, L.J. Methabolic syndrome, testosterone deficiency and erectile disfunction never come alone. Andrología 2008 40;4;259-264

7. Reilly MP, Rader DJ.. The metabolic syndrome: more than the sum of it's parts? Circulation. 2003;108:1546-51.

8. Sáez ME, Grilo A, Morón Fj, Manzano L, González-Pérez A, Serrano-Hernando A, Ruiz A,Ramírez-Lorca R, Serrano-Ríos M. The peroxisome proliferator-activated receptor delta gene is associated with obesity and interacts with the calpain 5 gene to modulate the phenotype. Cardiovascular Diabetology 2008 Jul 25;7:23.

9. Reaven GM, Chen Y-DI, Jeppesen J, Maheux P, Krauss RM. Insulin resistance and hyperinsulinemia in individuals with small, dense, low density lipoprotein particles. J Clin Invest 1993;92:141-6.

10. Ai A, Tanaka A, Ogita K, Sekine M, Numano F, Numano U, Reaven G. Relationship between hyperinsulinemia and remnant lipoprotein concentrations in patients with impaired glucose tolerance. J Clin Endocrinol Metabol 2000; 85:3557-60.

*11. Gimeno JA, Lou JM, Molinero E, Poned B, Portilla DP. Influencia del síndrome metabólico en el riesgo cardiovascular de pacientes con diabetes tipo 2. Rev Esp Cardiol 2004;57:507-13.

12. Alexander CM, Landsman PB, Teutsch SM, Haffner SM.. NECP-Defined metabolic syndrome, diabetes, and prevalence of coronary heart disease among NHANES III participants age 50 years and older. Diabetes. 2003;52:1210-4.

13. Ridker PM, Buring JE, Cook NR, Rifai N. C-reactive protein, the metabolic syndrome, and risk of incident cardiovascular events: a 8-year follow-up of 14,719 initially healthy American women. Circulation. 2003;107:391-7.

**14. Tsujimura A, Takada S, Matsuoka Y, Nakayama J, Takao T, Miyagawa Y, Sonoda M, Nishizawa H, Iwahashi H, Funahashi T, Nonomura N, Okuyama A. Adiponectin and testosterone in patients with symptoms of late-onset hypogonadism: is there a link? Int J Urol. 2009 Oct;16(10):830-5.

*15. Eikelis N, Schlaich M, Aggarwal A, Kaye D, Esler M.. Interactions between leptin and the human sympathetic nervous system. Hypertension. 2003;41:1072-9.

16. Chu NF, Spiegelman D. Plasma insulin, leptin and soluble TNF receptors levels in relation to obesity-related atherogenic and thrombogenic cardiovascular disease risk factors among men. Atheros- clerosis 2001;157: 495-503.

**17. Traish AM, Saad F, Feeley RJ, Guay A. The dark side of testosterone deficiency: III. Cardiovascular disease. JAndrol. 2009 Sep-Oct;30(5):477-94.

**18. Vela Navarrete, R.; Garcia Cardoso, J.V.; Pardo Montero, M.; Jimenez Mateos-Caceres, P.; Lopez Farré, A. Testosterona, función endotelial, salud cardiovascular y androgenodeficiencia del varón añoso. Arch. Esp. Urol. 2009; 62,3;173-178

19. Lopez Farré, A.; Macaya, C. Androgenodeficiencia y salud cardiovascular. Cuadernos de Urología 2009 56;16-22

*20. Daneshgari F., Brown J.S., Kusek J.W., Nyberg L.M. Urological complications of obesity and diabetes. J. Urol. 2009 182; S1-S56 (suplemento)

21. Brenner BM, Cooper ME, De Zeeuw D, Keane WF, Mitch WE, Parving HH, et al.. Effects of losartan on renal and cardiovascular outcomes in patients with type 2 diabetes and nephropathy. $\mathrm{N}$ Engl J Med. 2001;345:861-9.

*22. González-Juanatey JR, Mazón P, Soria F, Barrios V, Rodríguez L, Bertomeu V.. Actualización (2003) de las Guías de Práctica Clínica de la Sociedad Española de Cardiología en hipertensión arterial. Rev Esp Cardiol. 2003;56:487-97.

23. Sever PS, Dahlöf B, Poulter NR, Wedel H, Beevers G, Caulfield M, et al.. Prevention of coronary and stroke events with atorvastatin in hypertensive patients who have average or lower-than-average cholesterol concentrations in the Anglo-Scandinavian cardiac outcomes trial-lipid lowering arm (ASCOT-LLA): a multicentre randomised controlled trial. Lancet. 2004;361:1149-58.

*24. Makhsida N,Shah J,Yan G,Fisch H,Shabsigh R.Hypogonadism and metabolic syndrome: implications for testosterone therapy.J Urol Sep 2005; 174 (03):827-34.

25. Dandona P, Dhindsa S, Chaudhuri A, Bhatia V, Topiwala S, Mohanty P. Hypogonadotrophic hypogonadism in type 2 diabetes, obesity and the metabolic syndrome. Curr Mol Med.2008 Dec;8(8):816-28.

*26. Saad F, Gooren L, Haider A, Yassin A. An exploratory study of the effects of 12 month administration of the novel long-acting testosterone undecanoate on measures of sexual function and the metabolic syndrome.Arch Androl. 2007 NovDec;53(6):353-7.

**27. Zitzmann M. Testosterone deficiency, insulin resistance and the metabolic syndrome. Nat Rev Endocrinol. 2009 Dec;5(12):673-81.

28. Spark RF. Testosterone, diabetes mellitus, and the metabolic syndrome. Curr Urol Rep. 2007 Nov;8(6):467-71.

**29. Maggio M, Basaria S.Welcoming low testosterone as a cardiovascular risk factor. Int J Impot Res. 2009 Jul-Aug;21(4):261-4. 